\title{
RocS drives chromosome segregation and nucleoid occlusion in Streptococcus pneumoniae
}

\section{Chryslène Mercy ${ }^{\mathrm{a}}$, Jean-Pierre Lavergne ${ }^{\mathrm{a}}$, Jelle Slager ${ }^{\mathrm{b}}$, Adrien Ducret ${ }^{\mathrm{a}}$, Pierre Simon Garcia ${ }^{\mathrm{a}}$, Marie- Francoise Noirot-Gros ${ }^{\mathrm{c}}$, Nelly Dubarry ${ }^{\mathrm{a}}$, Julien Nourikyana ${ }^{\mathrm{a}}$, Jan-Willem Veening ${ }^{\mathrm{b}, \mathrm{d}}$, Christophe Grangeasse $^{\mathrm{a}, 1}$}

\author{
${ }^{a}$ Molecular Microbiology and Structural Biochemistry, UMR 5086, Université Claude Bernard Lyon 1, Centre National de la \\ Recherche Scientifique, 69367 Lyon, France \\ ${ }^{\mathrm{b}}$ Molecular Genetics group, Groningen Biomolecular Sciences and Biotechnology Institute, Centre for Synthetic Biology, \\ University of Groningen, 9747 AG, Groningen, The Netherlands \\ ${ }^{\mathrm{c}}$ Micalis institute, UMR1319, INRA, AgroParisTech, Université Paris-Saclay, 78350 Jouy-en-Josas, France and current address: \\ Biosciences Division, Argonne National Laboratory, Lemont, USA \\ ${ }^{\mathrm{d}}$ Department of Fundamental Microbiology, Faculty of Biology and Medicine, University of Lausanne, Biophore Building, $\mathrm{CH}-$ \\ 1015, Lausanne, Switzerland \\ ${ }^{1}$ Correspondence: c.grangeasse@ibcp.fr
}

\begin{abstract}
Segregation of replicated chromosomes in bacteria is poorly understood outside some prominent model strains and even less is known about how it is coordinated with other cellular processes. Here we report that RocS is crucial for chromosome segregation in the opportunistic human pathogen Streptococcus pneumoniae. RocS is membrane-bound and interacts both with DNA and the chromosome partitioning protein ParB to properly segregate the origin of replication region to new daughter cells. In addition, we show that RocS interacts with the tyrosineautokinase $\mathrm{CpsD}$ required for polysaccharide capsule biogenesis, which is crucial for $S$. pneumoniae's ability to prevent host immune detection. Altering the RocS-CpsD interaction drastically hinders chromosome partitioning and cell division. Altogether, this work reveals that RocS is the cornerstone of an atypical nucleoid occlusion system ensuring proper cell division in coordination with the biogenesis of a protective capsular layer.
\end{abstract}

\section{Introduction}

In dividing cells, accurate and faithful duplication and distribution of the genetic heritage are crucial steps toward the generation of viable and identical progeny. Unlike eukaryotes, in which chromosome segregation is performed by the well-known mitotic spindle (1), far less is known about the structures and mechanisms employed in bacteria. Intensive investigations have partly elucidated these processes in model bacteria like Escherichia coli, Bacillus subtilis and Caulobacter crescentus (for reviews see (2-5). However, these studies also highlight that there are large variations in the mechanisms involved, and it remains poorly understood in many other bacterial species.

This is the case for Streptococci and more specifically the opportunistic human pathogen Streptococcus pneumoniae (the pneumococcus), a prominent model to study the bacterial cell cycle (6). The pneumococcus is an ovoid-shaped bacterium that lacks some of the well-established systems controlling cell division, like the Min and the nucleoid occlusion systems (7). It possesses only the condensin SMC and an incomplete chromosome partitioning ParABS system, in which ParA is absent. Previous studies have evidenced that both ParB and SMC are involved, but not essential, in pneumococcal chromosome segregation (8). Notably, both individual or double deletion of parB and $s m c$ only lead to weak chromosome segregation defects, suggesting that other factors remain to be discovered. In line with this hypothesis, transcription was shown to contribute to pneumococcal chromosome segregation (9). The tyrosine-autokinase $\mathrm{CpsD}$ was also found to interfere with chromosome segregation (10). CpsD is primarily described as a key regulator of the export and synthesis of the polysaccharide capsule, the main virulence factor of the pneumococcus, that is exclusively produced at the pneumococcal division septum (1013). However, defective autophosphorylation of $\mathrm{CpsD}$ also generated elongated cells with an aberrant nucleoid morphology (10).

\section{Results and Discussion}

To further analyze the relationship between capsule production and chromosome biology, we screened a yeast two-hybrid genomic library of a pneumococcal laboratory strain (14) using CpsD as bait. A strong and reproducible interaction was identified with Spr0895, a protein with unknown function (Fig. S1A). The CpsD-Spr0895 interaction was confirmed biochemically using microscale thermophoresis (Fig. S1BC). The spr0895 gene is conserved among Streptococcaceae (Fig. S2) and is hereinafter referred to as rocS (Regulator of Chromosome Segregation) based on the observations we report below.

We first constructed a $\operatorname{rocS}$ deletion in the encapsulated virulent D39 strain and analyzed capsule production by immunofluorescence microscopy using anti-serotype 2 capsule antibodies (10). As observed for wild type cells, capsule was detected over the entire surface of $\Delta r o c S$ cells (Fig. 1A). In addition, immunodetection of the total fraction of capsule by western-blot revealed that capsule production and polymerization were not affected (Fig. S3). However, although the cell shape of $\triangle \mathrm{rocS}$ cells was not significantly altered, they displayed a growth defect with an increased generation time compared to wild type cells (Fig.S4). Surprisingly, when we looked at the DNA content of $\Delta r o c S$ cells using DAPI staining, we found that $13.9 \%$ of cells were 
A

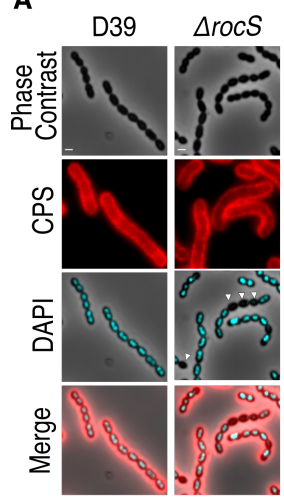

B

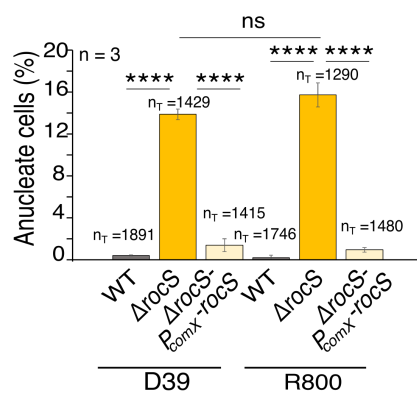

C

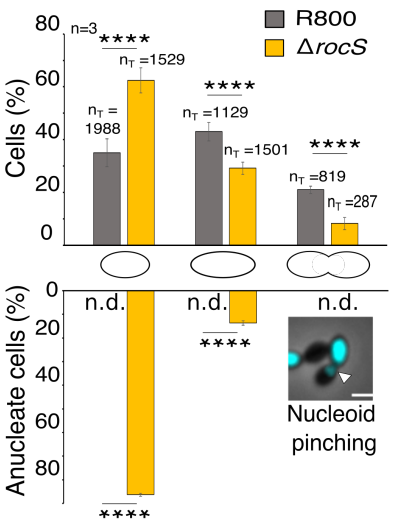

D

Normal nucleoid distribution

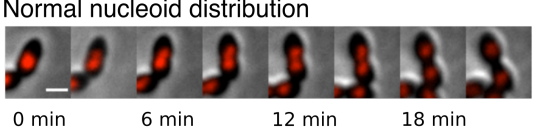

E

Absence of nucleoid segregation

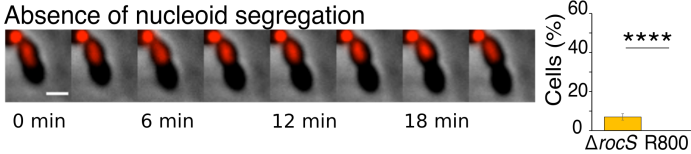

Fucleoid pinching

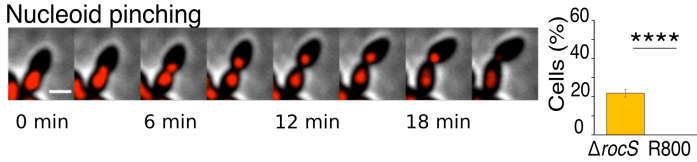

Fig. 1: Impact of rocS deletion on capsule production and nucleoid distribution

(A) Detection of capsular polysaccharides (CPS) and DNA in D39 and $\Delta$ rocS cells. Phase contrast (grey), CPS (red), DAPI (blue) and overlays are shown. Arrow heads indicate anucleate cells. (B) Percentage of anucleate cells in D39 and R800 (grey) strains, corresponding $\Delta$ rocS mutants (orange) and complemented strains (yellow). (C) Quantification of anucleate cells in the course of the cell cycle. R800 (grey) and $\Delta$ rocS (orange) cells were classified into three groups (nascent, elongated, constricting) according to the progression of the cell cycle. The top histogram shows the percentage of each group for each strain. The percentage of anucleate cells in each group is shown in the bottom histogram. Arrowheads indicate chromosome pinching in constricting cells. n.d.= none detected. In (B) and (C), standard deviations are indicated with error bars. (D-F) Still images from fluorescence time-lapse microscopy (Movies S1, S2 and S3) of WT (D) and $\Delta$ rocS cells (E and F) producing HIpA-mKate2. (D) Normal nucleoid segregation, (E) absence of nucleoid segregation and (F) nucleoid pinching. Histograms show the percentage of the nucleoid organization (absence or pinching) in WT and $\Delta$ rocS cells. In B-F, $n_{T}$ indicates the number of cells analyzed from 3 independent experiments and standard errors are indicated with error bars. (Two population proportions test: ${ }^{* * *} \mathrm{P}<0.0001$. ns $\mathrm{P}>0.5$ ). Scale bar, $1 \mu \mathrm{m}$.

anucleate (Fig. 1A-B). Unencapsulated and non-virulent laboratory R800 cells deficient for rocS showed similar growth defects and a comparable fraction of anucleate cells $(15.7 \%)$, indicating that these aberrant phenotypes were not dependent on capsule production (Fig. 1B and S5). Complementation of the $\Delta$ rocS in both R800 and D39 genetic backgrounds with an ectopic copy of $\operatorname{rocS}\left(\Delta \operatorname{rocS}-\mathrm{P}_{\text {comx }}-r o c S\right)$ restored the wild type phenotype with $1 \%$ and $1.5 \%$ of anucleate cells, respectively (Fig. 1B). By comparison, the deletion of well-established factors required for chromosome replication and/or segregation like ParB or SMC in S. pneumoniae, results in less than $4 \%$ and $2 \%$ of anucleate cells, respectively (10). Furthermore, we were unable to delete both $\operatorname{roc} S$ and either smc or parB, indicating that the deletion of roc $S$ is synthetically lethal with parB or $s m C$. Altogether, our results show that RocS has an important role in pneumococcal chromosome biology.

To understand the function of RocS, we analyzed $\Delta$ roc $S$ R800 cells at three different stages of the cell cycle (nascent, elongated and constricted cells, (Fig. 1C). By comparison with the relative proportion observed for wild type cells, we observed a significant increase of cells at the early stage of the cell cycle, relative to cells at the later stages: $62.5 \%$ of $\Delta$ roc $S$ cells displayed the typical morphology of rounded nascent cells while only $35 \%$ of wild-type cells harbored this morphology (Fig. 1C). Strikingly, a large majority of anucleate cells $(86.3 \%)$ were indeed observed at the early stage of the cell cycle (nascent cells). In addition, some cells at the later stages (constricted cells) of the cell cycle harbored asymmetric distribution of the DNA content, which suggests chromosome-pinching events (Fig. 1C).

To get more insight into chromosome dynamics in the absence of RocS, we performed time-lapse microscopy to image the nucleoid by localizing the pneumococcal histone-like protein, using a HlpA-mKate2 fusion (9). As expected, the chromosome duplicates at the early stage of the cell cycle and eventually splits into two parts that segregate to each daughter cell (Fig. 1D and Movie S1). In contrast, newly replicated chromosomes in $\Delta$ roc $S$ cells were either not segregated (7\%) (Fig. 1E and Movie S2), or partially segregated and eventually truncated by the newly forming septum $(21.8 \%)$, a process also known as the guillotine effect (15) (Fig. 1F and Movie S3). In the latter case, the signal of the truncated chromosome became diffuse and was ultimately degraded. In both cases, these aberrant chromosome-partitioning events led to the formation of anucleate nascent cells. To test if chromosome replication was affected in the R800 $\triangle r o c S$ mutant, we used qPCR to determine the ratio between the origin of replication (oriC) and the terminus region (ter) of the chromosome in exponentially growing cells (16) (Fig. S6). As expected, we observed that dividing wild type cells displayed a characteristic mean ratio of $1.68 \pm 0.28$ whereas this ratio was close to 1 for a thermo-sensitive $d n a A$ (the replication initiator protein) mutant shifted to non-permissive temperature. The origin-to-terminus ratios of $\Delta \operatorname{rocS}(1.67 \pm 0.24)$ and complemented $\Delta r o c S-P_{\text {com } x}-r o c S(1.56 \pm 0.24)$ cells were similar to that of wild type cells, indicating that RocS is not involved in chromosome replication. Together, our results show that chromosome segregation rather than chromosome replication is severely affected in the absence of RocS.

To characterize the contribution of RocS to chromosome segregation, we next examined the subcellular localization of the origin of replication (oriC) during the cell cycle of wild-type and $\Delta r o c S$ R800 cells (Fig. 2A-D). To visualize oriC at the single cell level, we engineered a system based on the ectopic production of a fluorescent fusion of RepC, the ParB homolog of Enterococcus faecalis, and insertion of $p a r S_{E f}$ sites from E. faecalis near the pneumococcal oriC (17) (Fig. 2A). Neither expression of repC-gfp nor insertion of $p a r S_{E f}$ sites influenced the pneumococcal cell cycle as evidenced by wild-type growth kinetics and cell morphology (Fig. S7). When produced, the RepC-GFP fusion, which binds specifically to the $\operatorname{par}_{E f}$ site, formed diffraction-limited foci in the vicinity of oriC, (Fig. 2B and Fig. S7). As previously characterized (18), oriC localized as a single focus located around mid-cell of nascent cells (Fig. 2B). The duplication of the focus was followed by rapid segregation of the two foci toward the center of 
A

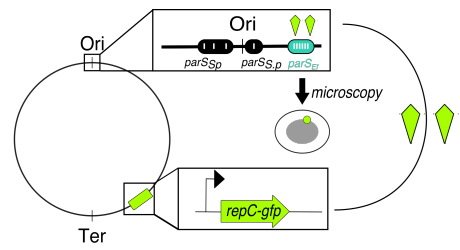

B
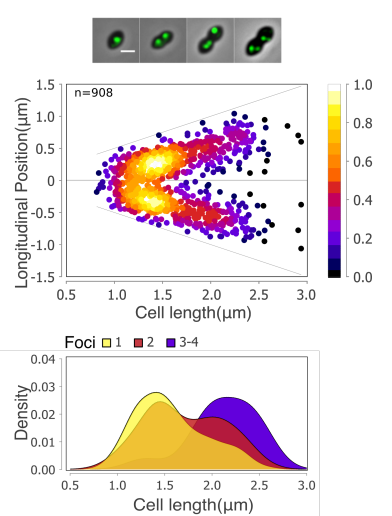

C

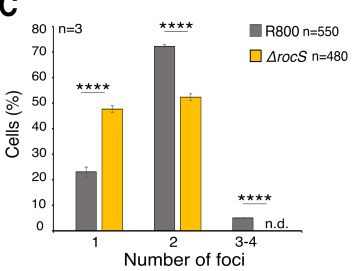

$\Delta$ rocs
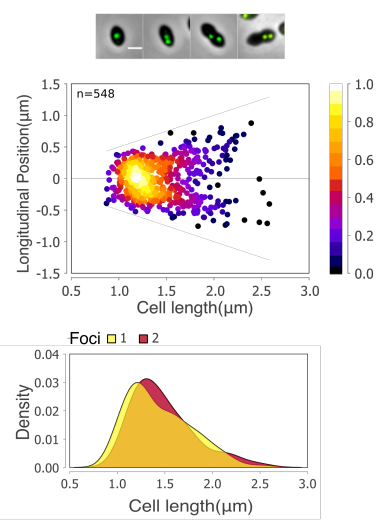

D

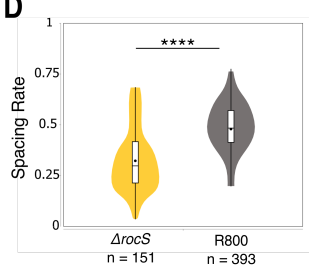

Fig. 2: oriC segregation patterns in wild-type and $\Delta$ rocS cells

(A) Schematic representation of the Par system used to image the origin of replication (oriC). pars sequences from $E$. faecalis ( $p a r S_{E f}$, blue oval) were inserted into the chromosome near the pneumococcal oriC while the parB homolog repC fused to gfp (RepC-GFP, green kite) is expressed ectopically under the control of the $P_{\text {comx }}$ promoter. Upon loading of GFP-RepC onto parS $S_{E f}$ sites, the localization of oriC is followed by fluorescence microscopy (green dot). par $S_{S p}$ indicates native pneumococcal parS sites. (B) (upper panels) Localization heat maps of oriC (GFP-RepC) positions along the cell length in wild-type and $\triangle$ rocS R800 cells. Representative overlays between phase contrast and GFP fluorescence signal of cells with either 1,2 or 3/4 foci are shown on the top. Scale bar, $1 \mu \mathrm{m}$. (lower panels) Kernel density plots of the cell length in relation to the number of foci in wild-type and trocS R800 cells. (C). Quantification of cells as a function of the number of oriC foci in WT (grey) and $\Delta$ rocS (orange) cells. Standard errors are indicated with error bars. (D). Measurements of the spacing rate (relative distance between 2 foci of oriC in relation to the cell length). $n_{T}$ indicates the number of cells analyzed. Experiments were performed in triplicates. (Two population proportions test: ${ }^{* * *} \mathrm{P}<$ 0.0001).

each daughter cell where they remain as the cell elongate. Interestingly, new cycles of chromosome segregation started early in the cell cycle, even before the completion of division, as attested by the $4.5 \%$ of nascent cells containing 2 foci and the $5 \%$ of cells at the later stage of the cell cycle containing 3 or 4 foci (Fig. 2B and 2D). By comparison, the subcellular localization of oriC throughout the cell cycle was strongly affected in the absence of RocS. After duplication, most of the two foci remained near mid-cell and did not segregate toward the opposite poles as the cells elongate (Fig. 2C). On average, the spacing rate (relative distance between 2 foci of oriC in relation to the cell length) was significantly lower in $\Delta r o c S$ cells $(0.32 \pm 0.003)$ than in WT

cells $(0.47 \pm 0.003)$ (Fig. 2E). Furthermore, the proportion of cells with single foci was significantly higher in $\Delta \operatorname{rocS}$ cells $(47.6 \%)$ than in wildtype cells $(23 \%)$. Since chromosome replication was not affected in $\Delta r o c S$ cells (Fig. S6), this observation suggests that after replication, some oriC copies may be too close to be detected as separated foci in $\Delta \operatorname{roc} S$ cells. Finally, we did not detect constricting cells containing 3 or 4 foci in $\Delta \operatorname{rocS}$ cells (Fig. 2C and 2D). Altogether, these data show that the two newly replicated chromosome origins segregate less efficiently in the absence of RocS, reflecting its crucial role in chromosome segregation.

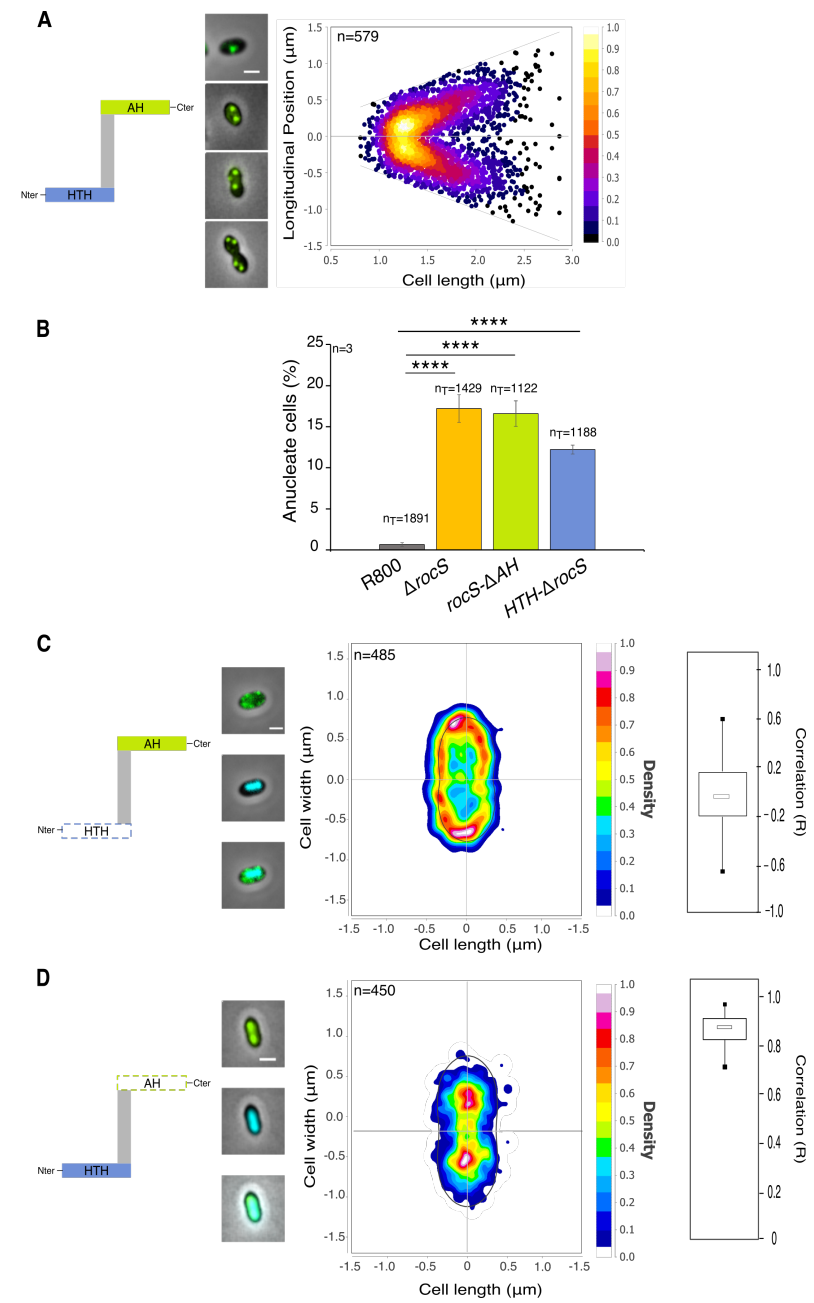

Fig. 3. Localization of GFP-RocS and derivatives and impact on nucleoid localization

Schematic representations of RocS and derivatives are shown on the left of panels $A, C$ and $D$. (A) Heat map representing the longitudinal localization of GFP-RocS as a function of the cell length in R800 cells. Representative overlays of cells with either 1,2 or $3 / 4$ foci are shown on the left. (B) Histogram showing the percentage of anucleate cells for rocS- $\triangle A H$ and $\triangle H T H$-rocS R800 strains. Standard errors are indicated with error bars. $\mathrm{n}_{\mathrm{T}}$ indicates the total number of cells analyzed from three independent experiments. (Two population proportions test: $* * * *$ $P<0.0001$ ). (C-D) Heat map representing the 2-dimensional localization patterns of GFP- $\Delta \mathrm{HTH}$-RocS (C) and GFP-RocS- $\Delta$ AH (D) in R800 cells. Representative overlays of phase contrasts and, GFP or DAPI fluorescence signals, or both signals, are shown on the left of the heat maps. Scale bar, $1 \mu \mathrm{m}$. The distribution of the Pearson correlation coefficient (R), measured between the DAPI and GFP signals for each strain are shown as box and whisker plots on the right. 
We next followed the subcellular localization of RocS during the cell cycle using a reporter strain expressing a fluorescent fusion of RocS. The gene $g f p$-rocS replaces the rocS gene at the native chromosomal locus and encodes a largely functional fusion protein, as attested by wild-type growth kinetics, cell morphology, intracellular level and low level of anucleate R800 cells (3\%) (Fig. S8 and S9).

The GFP-RocS fusion protein formed faint diffraction-limited foci that were mobile, able to both fuse into stationary and brighter foci and to re-segregate (Movie S4). Interestingly, while mobile foci showed no specific localization during the cell cycle, the brighter foci showed a dynamic reminiscent of that of oriC. Indeed, brighter foci mostly localized around mid-cell of nascent cells and positioned toward the center of the daughter cell as the cell elongates (Fig. 3A). Bioinformatic analysis of the RocS sequence predicted the presence of a C-terminal membrane-binding amphipathic helix (AH) homologous to that of MinD of Escherichia coli (19) and an N-terminal helix-turn-helix domain (HTH, InterPro IPR000047) characteristic of DNA-binding proteins (20) (Fig. S10). These two domains are required for the function of RocS in chromosome segregation as both $\triangle H T H$-rocS and rocS- $\triangle A H \mathrm{R} 800$ cells displayed growth and viability defects as well as an anucleate phenotype similar to $\Delta r o c S$ R 800 cells (Fig. 3D and Fig. $\mathrm{S} 11)$. In addition, deletion of either the AH or the HTH domains drastically altered the localization pattern of RocS (Fig. 3B-C). The deletion of the $\mathrm{N}$ - terminal HTH domain resulted in the discontinuous redistribution of GFP- $\triangle H T H-R o c S$ at the cell periphery. On the other hand, GFP-RocS- $\triangle \mathrm{AH}$ displayed a diffused localization in the pneumococcal cell, which co-localized with the nucleoid (median $\mathrm{R}$ $=0.85$, interquartile range $=0.83-0.92$ ) (Fig. 3C). Using gel shift assays, we showed that RocS- $\triangle \mathrm{AH}$ binds directly to DNA (Fig. S12). This DNA binding was independent of the length, the GC content or the sequence of the tested DNA. Altogether, these data show that the Cterminal $\mathrm{AH}$ is required for the interaction of RocS with the membrane, while the N-terminal HTH domain mediates RocS DNA binding. Collectively, these data show that the interactions of RocS with both the chromosome and the membrane are essential for its function in chromosome segregation.

We finally questioned the biological role of the interaction between RocS and the tyrosine-autokinase CpsD (Fig. S1). Previous findings showed that CpsD possesses a structural fold comparable to that of ParA proteins that usually assist ParB in chromosome segregation (10, $(21,22)$. Since ParA is absent in the pneumococcus (7) and CpsD interacts directly with $\mathrm{ParB}$, it was proposed that $\mathrm{CpsD}$ could act as a ParA-like protein (10). Interestingly, this interaction is modulated by the autophosphorylation of CpsD: mimicking permanent phosphorylation of $\mathrm{CpsD}(\mathrm{CpsD}-3 \mathrm{YE})$ promotes capsule biogenesis and normal chromosome segregation by enabling ParB mobility (10) (Fig. 4A). By contrast, defective autophosphorylation of CpsD (CpsD-3YF) not only impairs capsule production, but also reduces ParB mobility, inducing aberrant chromosome segregation and leading to cell elongation (10) (Fig. 4B). By consequence, even in the absence of a conserved nucleoid occlusion system in the pneumococcus (7), cell division seems inhibited to protect the nucleoid against truncation by the newly forming septum when CpsD is not phosphorylated. Interestingly, we also demonstrated that RocS interacts with ParB both in vivo and in vitro (Fig. S13). Therefore, we wondered if this cell division block was due to RocS. To test this hypothesis, we deleted rocS in D39 strains mimicking either permanent or defective phosphorylation of $\mathrm{CpsD}$ (respectively $\Delta r o c S-c p s D-3 Y E$ and $\Delta r o c S-c p s D-3 Y F$ ) and looked at the cell morphology, capsule production and DNA content. While deletion of $\operatorname{rocS}$ in the permanent phosphorylation $\operatorname{cps} D-3 Y E$ mutant did not impact the cell morphology, the deletion of rocS suppressed the elongated phenotype of the defective phosphorylation cpsD-3YF mutant (Fig. 4A). In both cases, the deletion of rocS is accompanied by approximately $13 \%$ of anucleate cells $(12.8 \%$ and $13.7 \%$ respectively). The deletion of $\operatorname{roc} S$ in the phospho-ablative mutant therefore abrogates the cell division block. Non-phosphorylated CpsD together with RocS could therefore be viewed as a bona fide nucleoid occlusion system.
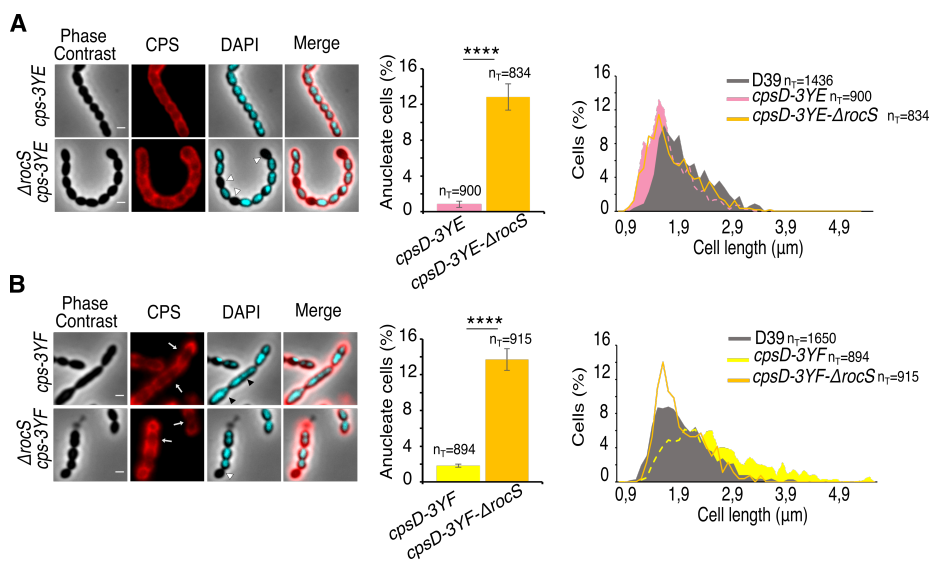

Fig. 4. Deletion of rocS in phospho-ablative and phospho-mimetic CpsD mutants

Detection of CPS and DNA in (a) $c p s D-3 Y E$ and $c p s D-3 Y E-4 r o c S$ and (b) $c p s D$ $3 Y F$ and $c p s D-3 Y F-\Delta r o c S$. Phase contrast (grey), CPS (red), DAPI (blue) and overlays are shown on the left. White arrows show CPS production defects, white arrowheads show anucleate cells and black arrowheads show nucleoid segregation defects. Scale bar, $1 \mu \mathrm{m}$. The corresponding percentage of anucleate cells are shown in the middle. (Two population proportions test: $* * * * \mathrm{P}<$ $0.0001)$. The corresponding distribution of the cell length are shown on the right. $\mathrm{n}_{\mathrm{T}}$ indicates the number of cells analyzed from 3 independent experiments and standard errors are indicated with error bars.

Typical nucleoid occlusion systems prevent the assembly of the FtsZ ring over the nucleoid. However, the FtsZ-ring is properly positioned in elongated cells (10) indicating that cell constriction, but not FtsZ-ring assembly, is blocked in the cps $D$ phospho-ablative mutant. As the deletion of $\operatorname{roc} S$ alleviates this phenotype, one can further conclude that RocS is the cornerstone of a new type of nucleoid occlusion system that prevents the constriction of the FtsZ-ring rather than impeding its assembly (Fig. 5). Which exact step of cell septation is blocked by the here-identified nucleoid occlusion system remains unclear, but it is tempting to speculate that RocS could work in partnership with the major cell division regulator of the pneumococcus, the eukaryotic-like protein-kinase StkP, whose inactivation also results in cell elongation.

$(23,24)$. In any case, this work illustrates that pathways controlling chromosome segregation and cell division are far more diverse than expected and suggests that the RocS system is likely valid for all Streptococcaceae (Fig. S2). The "raison d'être" of such a regulatory process coordinating capsule synthesis with cell cycle progression is likely to make sure that cells are covered by capsule at every step of the cell cycle in order to prevent detection by the human immune system. 


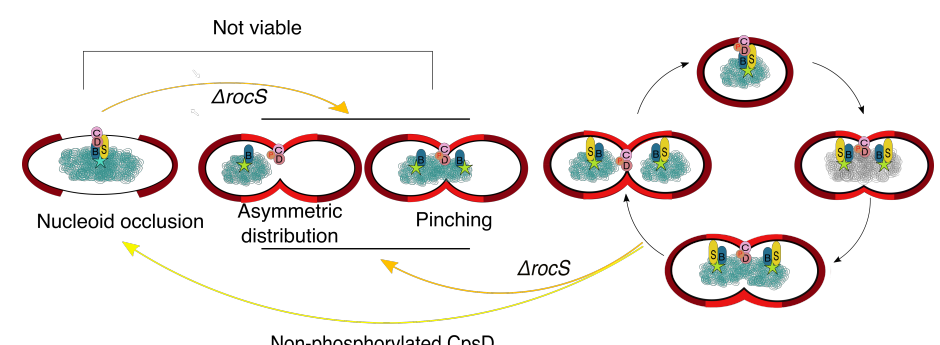

Non-phosphorylated CpsD

Figure 5: Model for the nucleoid occlusion system coordinating capsule synthesis, chromosome segregation and pneumococcal cell division

When CpsD is autophosphorylated, capsule is properly produced and RocS and ParB actively contribute to chromosome segregation (viable encapsulated progeny). By contrast, non-phosphorylated CpsD hinders both capsule synthesis and chromosome segregation inducing a division block. The deletion of rocS alleviates the division block and results in uncontrolled cell constriction with improper chromosome segregation (pinching and asymmetric distribution) leading to non-viable progeny. ParB, RocS, CpsD and its transmembrane activator CpsC are indicated by blue, yellow, brown and pink circles, respectively. Red "P" and the turquoise star indicate CpsD autophosphorylation and the oriC region, respectively. Capsule is shown in light (new capsule produced during cell division) and dark (inherit from the mother cell) red.

\section{Materials and Methods}

\section{Strains and growth conditions}

Strains used in this study are listed in the Table S1. Streptococcus pneumoniae R800 and D39 and derivatives were cultivated at $37^{\circ} \mathrm{C}$ in $\mathrm{C}+\mathrm{Y}$ medium or Todd-Hewitt Yeast (THY) broth.

Cell growth curves were monitored in JASCO V-630-BIOspectrophotometer and the optical density was read automatically every 10 min. Escherichia coli XL1-B strain (25) was used for cloning and $E$. coli BL21 (26) for overproduction of $\mathrm{CpsC} / \mathrm{D}$, RocS, RocS- $\triangle \mathrm{AH}$ and ParB. E. coli strains were grown in Luria Bertani broth (LB) supplemented with appropriate antibiotic. Growth was monitored by optical density (OD) readings at $550 \mathrm{~nm}$ or $600 \mathrm{~nm}$ for $S$. pneumoniae or E. coli strains, respectively.

\section{Construction of plasmids and strains}

Gene modifications ( $g f p$ and flag fusions, knock-out and domain deletion) in $S$. pneumoniae were achieved by homologous recombination using the two-step procedure based on a bicistronic kanrpsL cassette called Janus (27) and constructed at their native chromosomal locus. They are thus expressed under the control of the native promoter and represent the only source of protein.

$\Delta$ rocS D39 and $\Delta$ rocS R800 strains were complemented ectopically for rocS expression using the strategy described by (28) using the competence inducible system of Streptococcus thermophilus. The ComS-inducible comR DNA fragment was introduced between the treR and $a m i F$ loci of both strains. Then, the rocS copy under the control of the $\operatorname{com} X$ promoter was inserted between the cps $N$ and cps $O$ genes in $\mathrm{R} 800$ or at the bgaA locus in D39 strains.

For constructing the system for tagging ori, we used the parS sites and the ParB homologue RepC fused to the GFP from Enteroccocus faecalis (17). The parS sites were inserted between thmA and IS1167 loci near the pneumococcal origin of replication. Then, the repC-gfp under the control of the promoter of the comX gene of Streptococcus thermophilus were used by PCR and inserted between the $c p s N$ and $c p s O$ genes in the $\mathrm{R} 800$ strain. repC-gfp expression was induced with $5 \mu \mathrm{M}$ ComS.
To construct the thermo-sensitive dnaA R800 mutated strain, we PCR amplified the dnaA(T1193C) mutated gene of the D39 thermo-sensitive mutant described in (9). The DNA fragment was then transformed in the R800 strain and cells were plated at $30^{\circ} \mathrm{C}$. After overnight growth, colonies were resuspended in THY and cultured again on plates at either $30^{\circ} \mathrm{C}$ or $40^{\circ} \mathrm{C}$. The mutation in dnaA was checked by DNA sequencing in clones growing at $30^{\circ} \mathrm{C}$ but not at $40^{\circ} \mathrm{C}$.

For the construction of the plasmid overexpressing RocS- $\Delta \mathrm{AH}-6 \mathrm{His}$, we PCR amplified a DNA fragment coding for RocS from Met1 to Gln150 using chromosomal DNA from $S$. pneumoniae R800 as template. The obtained DNA fragment was cloned between the NdeI and PstI cloning sites of pT7-7 (29). The other plasmids used in this study are described in Table $\mathrm{S} 1$.

The oligonucleotides used for all construction are listed in Table S2. Plasmids and pneumococcal strains were verified by DNA sequencing to verify error-free PCR amplification.

\section{Protein purification}

Purification of the chimera 6His-CpsC/D and ParB-6His was performed as described previously (10). To purify RocS- $\triangle \mathrm{AH}-6 \mathrm{His}$, E. coli BL21 were used and cultured at $37{ }^{\circ} \mathrm{C}$ in $\mathrm{LB}$ medium. At $\mathrm{OD}_{600}=0.6,1 \mathrm{mM}$ IPTG was added and ells culture were incubation was continued for $3 \mathrm{~h}$. at $37{ }^{\circ} \mathrm{C}$. Cells were then harvested by centrifugation and resuspended in buffer A (Tris-Hcl $25 \mathrm{mM}, \mathrm{pH} 7.5 ; \mathrm{NaCl} 1 \mathrm{M}$, imidazole $10 \mathrm{mM}$; glycerol $10 \%$ ) containing $10 \mathrm{mg} \mathrm{mL}^{-1}$ of lysozyme, $1 \mu \mathrm{g} \mathrm{ml}^{-1}$ of protease inhibitor (Roche Diagnostics). After sonication and centrifugation, the supernatant was loaded on to a Ni-NTA agarose resin (Qiagen) and extensively washed with buffer A containing $20 \mathrm{mM}$ imidazole. RocS-6His was eluted with buffer B (Tris-Hcl $25 \mathrm{mM}, \mathrm{pH}$ 7.5; $\mathrm{NaCl} 300 \mathrm{mM}$, imidazole $300 \mathrm{mM}$; glycerol 10\%). Pure fractions were pooled and dialyzed against buffer C (HEPES $50 \mathrm{mM}, \mathrm{pH} 7.5$ or Tris $\mathrm{pH} 7,525 \mathrm{mM}$; $\mathrm{NaCl} 150 \mathrm{mM}$, glycerol 10\%). Protein concentrations was determined using a Coomassie assay protein dosage reagent (Uptima).

\section{Co-immunoprecipitation and immunoblot analysis}

For co-immunoprecipitation, cultures of $S$. pneumoniae cells were grown at $37^{\circ} \mathrm{C}$ in $\mathrm{C}+\mathrm{Y}$ medium until $\mathrm{OD}_{550 \mathrm{~nm}}=0.3$. Cells pellets were incubated at $30^{\circ} \mathrm{C}$ for $30 \mathrm{~min}$ in buffer A (Tris- $\mathrm{HCl} 0.1 \mathrm{M}, \mathrm{pH} 7.5$; $\mathrm{MgCl}_{2} 2 \mathrm{mM}$, Sucrose $1 \mathrm{M}, 6 \mathrm{mg} \mathrm{mL}^{-1}$ of DNase I and RNase A, $1 \mu \mathrm{g}$ $\mathrm{ml}^{-1}$ of protease inhibitor). After centrifugation at $4{ }^{\circ} \mathrm{C}$, the pellet was resuspended in buffer B (Tris-Hcl $0.1 \mathrm{M}, \mathrm{pH} 7.5$; EDTA $1 \mathrm{mM}, 0.1 \%$ Triton, $6 \mathrm{mg} \mathrm{mL}^{-1}$ of DNase I and RNase A, $1 \mu \mathrm{g} \mathrm{ml}^{-1}$ of protease inhibitor) and incubated $15 \mathrm{~min}$ at room temperature before being harvested by centrifugation. The supernatant was then incubated with Dynabeads (Invitrogen) coupled with $20 \mu \mathrm{g}$ anti-Flag antibodies and incubated for 2 hour at $4{ }^{\circ} \mathrm{C}$. After extensive wash with buffer $\mathrm{C}$ (TrisHcl $10 \mathrm{mM}, \mathrm{pH} 7.5$, EDTA $0.5 \mathrm{mM}, 0.1 \%$ Triton, $\mathrm{NaCl} 150 \mathrm{mM}, 1 \mu \mathrm{g}$ $\mathrm{ml}^{-1}$ of protease inhibitor), Protein-bounded bead were eluted with SDS-PAGE loading buffer at $95^{\circ} \mathrm{C}$ for $10 \mathrm{~min}$ and analyzed by SDSPAGE and immunoblotting using a rabbit anti-GFP antibody at $1 / 10,000$ (AMS Biotechnology) or the anti-FLAG antibody at $1 / 1,000$ (Sigma). For immunoblot analysis, $S$. pneumoniae pellets were resuspended in TE-buffer ( $25 \mathrm{mM}$ Tris- $\mathrm{HCl} \mathrm{pH} 7.5,1 \mathrm{mM}$ EDTA) supplemented with protease and phosphatase inhibitor cocktail II (Sigma-Aldrich) and opened by sonication. $25 \mu \mathrm{g}$ of crude extracts were analyzed by SDS-PAGE, electrotransferred onto a polyvinylidene difluoride membrane and incubated with either rabbit anti-RocS at $1 / 5,000$ (produced by Eurogentec with purified RocS- $\Delta \mathrm{AH}-6 \mathrm{His}$ ), rabbit-anti-enolase polyclonal antibody at $1 / 50,0000$ (23) or rabbit anti- 
serotype 2 CPS polyclonal antibody at 1/2,000 (Statens serum Institute). A goat anti-rabbit polyclonal antibody horseradish peroxidase (HRP) conjugated (Biorad) was used at 1:5000 to reveal immunoblots.

\section{Yeast-two hybrid}

The yeast two hybrid genetic screens were carried out using a mating strategy as described previously (14) (30). Construction of the pGBDUcpsD bait plasmid and expressing CpsD fused to the DNA-binding domain of Gal4 (BD) was described in (10). This plasmid was introduced in the PJ69-4( $\alpha$ ) haploid strain. This strain was then mated with PJ69-4 haploid $(\alpha)$ strain harboring a library of pGAD plasmids expressing genomic fragments of $S$. pneumonaie R6 in fusion with the GAL4 activating domain (AD) (14). Potential binary interactions were selected by the ability of the yeast diploids to grow on synthetic media agar SC-LUH lacking Leucine (L) and Uracil (U) to select for maintenance of plasmids pGAD and pGBDU, respectively, as well as histidine $(\mathrm{H})$, to selects for the interaction (31). Additionally, binary interactions were tested by a matrix-based approach by mating haploid cells expressing BD-CpsD, with haploid cells of complementary mating type expressing the AD-prey protein fusions $\operatorname{RocS}_{50-163}$, RocS, CpsC and CpsD. Diploids were first selected onto $-\mathrm{LU}$ media and further tested for interacting phenotypes (i.e. ability to grow on SC-LUH selective agar plates) to reveal binary interactions between bait and prey proteins.

\section{Preparation and analysis of CPS}

CPS were prepared as previously described (10). Briefly, S. pneumoniae cultures were grown until $\mathrm{OD}_{550 \mathrm{~nm}}=0.3$, washed once with PBS and resuspended in buffer A (Tris-HCL 50nM, pH 7.4; sucrose 20\%; $\mathrm{MgSO}_{4} 50 \mathrm{nM}$ ). The solution was then added with 400 units of mutanolysin and $6 \mu \mathrm{g} / \mu \mathrm{l}$ of DNase and RNase and incubated overnight at room temperature. After centrifugation at $16,000 \mathrm{xg}$ for $20 \mathrm{~min}$ at 4 ${ }^{\circ} \mathrm{C}$, pellets were resuspended in the same volume of buffer A. $10 \mu \mathrm{L}$ of the mixture were then mixed with $5 \mu \mathrm{l}$ of buffer B (Tris-HCl $50 \mathrm{mM}$, pH 8.0; EDTA $50 \mathrm{mM}$; Tween20 0.5\%; Triton X100 0.5\%) and $20 \mu \mathrm{g}$ of proteinase $\mathrm{K}$, incubated $30 \mathrm{~min}$ at $37^{\circ} \mathrm{C}$ and analyzed by SDS-PAGE and immunoblotting.

\section{Microscopy techniques}

Cells were grown until $\mathrm{OD}_{550 \mathrm{~nm}}=0.1$. For immunofluorescence microscopy, cells were mixed with the rabbit-serotype 2 CPS polyclonal antibody (Statens Serum Institute) at $1 / 1,000$, washed and then incubated with the anti-rabbit Dylight-549 antibody (Jackson ImmunoResearch) at 1/2,000. After a last wash with PBS, CPS were imaged.

For DAPI staining, $10 \mu 1$ of $S$. pneumoniae cell culture were mixed with $1 \mu \mathrm{l}$ of DAPI at $2 \mu \mathrm{g} / \mu \mathrm{l}$ (Molecular Probes) and incubated $5 \mathrm{~min}$ at room temperature. For mkate 2 and GFP fluorescence imaging, cells were spotted on pads made of $1.5 \%$ agarose in $\mathrm{C}+\mathrm{Y}$ medium at $37^{\circ} \mathrm{C}$ as described in (32). Slides were visualized with a Nikon TiE microscope fitted with an Orca-CMOS Flash4 V2 camera with a $100 \times 1.45$ objective. Images were collected using NIS-Elements (Nikon). Images were analyzed using the software ImageJ (http://rsb.info.nih.gov/ij/) and the plugin MicrobeJ (33).

Diffraction-limited foci of RepC-GFP or GFP-RocS were detected using the feature/spot detection option in MicrobeJ. This option combines spatial 2D filtering and 2D local maxima algorithm to localize single fluorescent maxima in each detected cell. Each maximum was then fit to a single peak or a multi peak 2D Gaussian curve, to determine their amplitude, their FWHM (Full width at half Maximum) and their coordinates at the subpixel resolution. Maxima were finally filtered based on the goodness of the fit and their amplitude. Their subcellular localizations were automatically computed for each associated particle.

\section{Microscale thermophoretic analysis}

Microscale thermophoresis was used to test the interaction of RocS-AH with the chimeras $\mathrm{CpsC} / \mathrm{D}$ and ParB (34). Binding experiments were carried out with a Monolith NT.115 Series instrument (Nano Temper Technologies GMBH). RocS- $\triangle \mathrm{AH}$ was labeled with the red dye NT647 . Briefly, sample containing $50 \mathrm{nM}$ of labeled RocS- $\Delta \mathrm{AH}-6 \mathrm{His}$ and increasing concentrations of 6 His-CpsC/D (from $275 \mathrm{pM}$ to 9 $\mu \mathrm{M}$ ) or ParB-6His (from $427 \mathrm{pM}$ to $14 \mu \mathrm{M}$ ) were loaded on $\mathrm{K} 023$ Monolith NT.115 hydrophobic capillaries and thermophoresis was measured for $30 \mathrm{~s}$ at $25^{\circ} \mathrm{C}$. Each measurement was made in triplicates. Experiments were carried out at $25^{\circ} \mathrm{C}$ in $10 \mathrm{mM}$ HEPES pH $7.5,150 \mathrm{mM}$ $\mathrm{NaCl}$ and $0.05 \%$ Tween-20. Analysis was performed with the Monolith software. Affinity $\mathrm{KD}$ was quantified by analyzing the change in normalized fluorescence (Fnorm $=$ fluorescence after thermophoresis/initial fluorescence) as a function of the concentration of the titrated $6 \mathrm{His}-\mathrm{CpsC} / \mathrm{D}$ or ParB-6His proteins.

\section{oriC-ter Ratio determination by real-Time qPCR}

DNA genomic was extracted using the DNA maxima Kit (Qiagen). Real-time qPCR was performed as described previously (16). Briefly, each $20 \mu \mathrm{l}$ sample consisted of $8.8 \mathrm{ng}$ of DNA, $0.6 \mathrm{pmol}$ of each primer (Table S2), and $10 \mu 1$ of the 2x SYBR Green Supermix (Bio-Rad). Amplification was performed on an iQ5 Real-Time PCR Detection System (Bio-Rad). To find amplification efficiencies, Monte Carlo simulations were performed in $\mathrm{R}$. Average $\mathrm{C}_{\mathrm{t}}$-values and their corresponding standard deviations were used to simulate 10,000 new sets of $\mathrm{C}_{\mathrm{t}}$-values that were used to compute the amplification efficiencies for each set. From that population of possible efficiencies, averages and standard deviations were derived. Analysis of the realtime qPCR experiments for oriC-ter ratio determination was performed using the $2^{-\triangle \triangle C T}$ method (35), with the important difference that the earlier found amplification efficiencies were used to determine the foldchange per cycle, instead of assuming it to equal 2. As a reference, cells with an assumed oriC-ter ratio of 1 were used. For that, a thermosensitive DnaA-mutant (M398T) was grown at $30^{\circ} \mathrm{C}$ until an $\mathrm{OD}_{600}$ of 0.05 . Then, cells were transferred to non-permissive temperature $\left(40^{\circ} \mathrm{C}\right)$ and incubated for 1 hour, followed by harvesting and isolation of chromosomal DNA. Uncertainties in oriC-ter ratios were also determined by Monte Carlo simulations.

\section{Bioinformatic analyses}

For the phylogenetic analysis, homologues of RocS were retrieved using iterative BLASTP from BLAST package 2.2.6 against a local database containing 4466 prokaryotic complete proteomes retrieved from NCBI ftp (ftp://ftp.ncbi.nlm.nih.gov/). The Spr0895 amino acid sequence (NP_358489.1) was used as first seed. Protein sequences detected as homologues were aligned with MAFFT v7.123b (36) and used to build an HMM profile with HMMER v3.1b1 (37). The profile was then used to query the local database with HMMSEARCH from the HMMER package. Plasmidic sequences have been removed from the analysis. Phylogeny of Lactobacillales has been inferred from a supermatrix of ribosomal proteins. One strain per family was selected to represent each family in Lactobacillales and a sequence of one species 
of Listeriaceae was added to root the tree. The sequences were aligned using MAFFT (L-INS-I option) and trimmed with BMGE-1.1 (option BLOSUM30) (38). The evolution model was chosen using BIC criteria and the phylogeny was inferred using PhyML (39) $(\mathrm{LG}+\mathrm{I}+\mathrm{F}+\mathrm{G} 4,8$ sequences, 6219 positions).

Secondary structure predictions of RocS were obtained using PSIPRED (40). The helical representation of RocS and MinD of Escherichia coli was made using http://www.tcdb.org/progs/?tool=pepwheel.

\section{Electrophoretic mobility shift assay (EMSA)}

EMSA were carried out by incubating different concentrations of purified protein RocS- $\Delta \mathrm{AH}-6 \mathrm{His}(0 ; 5 ; 10 ; 15 \mu \mathrm{M})$ with 50 ng of DNA in the following buffer $\left(500 \mathrm{mM}\right.$ Tris- $\left.\mathrm{HCl} \mathrm{pH} 8.8,50 \mathrm{mM} \mathrm{MgSO}_{4}\right)$. DNA fragments of different length and percentage of GC content were PCR amplified (pUC18, gfp or genomic DNA of Pseudomonas aeruginosa PA7) using primers listed in Table S2. Reactions were incubated for $15 \mathrm{~min}$ at $37{ }^{\circ} \mathrm{C}$ before being loaded on $1 \%$ agarose gels. Gels were stained with ethidium bromide and revealed with UV light.

\section{Author Contributions}

C.G. directed the study. C.M. conducted the experiments of cell biology, genetics with J.N., protein purification and western blot analysis. C.M. and A.D. performed image analyses. C.M. and N.D. implemented the Ori localization system. J.P.L. performed microscale thermophoresis experiments and contributed to protein purification. C.M. and J.S. performed oriC/ter ratio experiments. M.F.N.G. performed yeast twohybrid experiments. P.S.G. performed phylogeny analyses. All authors designed and analyzed the data. C.G. and J.W.V. wrote the manuscript and all authors edited the manuscript. The authors declare no conflict of interest.

\section{Acknowledgments}

Work on the Grangeasse lab is supported by grants from the CNRS, the University of Lyon, the Agence National de la Recherche (ANR-10BLAN-1303-01 and ANR-15-CE32-0001-01), the Region AuvergneRhône-Alpes (financial support for C.M. and P.S.G.), the Fondation pour la Recherche Médicale (financial support for N.D. (ING20150532637) and C.M. (FDT20170437272)) and the BettencourtSchueller Foundation. Work in the Veening lab is supported by the Swiss National Science Foundation (project grant 31003A 172861), a VIDI fellowship (864.11.012) of the Netherlands Organization for Scientific Research (NWO-ALW), a JPIAMR grant (50-52900-98-202) from the Netherlands Organization for Health Research and Development (ZonMW) and ERC starting grant 337399-PneumoCell. We thank Stéphanie Ravaud for help in RocS structural predictions and Keith Weaver (University of South Dakota) for providing us with the pAD1 plasmid. We acknowledge the contribution of the Protein Science facility of the "SFR Biosciences Gerland-Lyon Sud (UMS344/US8)".

\section{This article contains supporting information online.}

\section{References}

1. C. E. Walczak, R. Heald, Mechanisms of mitotic spindle assembly and function. Int Rev Cytol 265, 111-158 (2008).

2. E. Toro, L. Shapiro, Bacterial chromosome organization and segregation. Cold Spring Harb Perspect Biol 2, a000349 (2010).
3. R. Reyes-Lamothe, E. Nicolas, D. J. Sherratt, Chromosome replication and segregation in bacteria. Annu Rev Genet 46, 121143 (2012).

4. X. Wang, P. Montero Llopis, D. Z. Rudner, Organization and segregation of bacterial chromosomes. Nat Rev Genet 14, 191-203 (2013).

5. A. Badrinarayanan, T. B. Le, M. T. Laub, Bacterial chromosome organization and segregation. Annu Rev Cell Dev Biol 31, 171-199 (2015).

6. C. Grangeasse, Rewiring the Pneumococcal Cell Cycle with Serine/Threonine- and Tyrosine-kinases. Trends Microbiol 24, 713724 (2016).

7. M. G. Pinho, M. Kjos, J. W. Veening, How to get (a)round: mechanisms controlling growth and division of coccoid bacteria. Nat Rev Microbiol 11, 601-614 (2013).

8. A. Minnen, L. Attaiech, M. Thon, S. Gruber, J. W. Veening, SMC is recruited to oriC by ParB and promotes chromosome segregation in Streptococcus pneumoniae. Mol Microbiol 81, 676-688 (2011).

9. M. Kjos, J. W. Veening, Tracking of chromosome dynamics in live Streptococcus pneumoniae reveals that transcription promotes chromosome segregation. Mol Microbiol 91, 1088-1105 (2014).

10. J. Nourikyan et al., Autophosphorylation of the Bacterial TyrosineKinase CpsD Connects Capsule Synthesis with the Cell Cycle in Streptococcus pneumoniae. PLoS Genet 11, e1005518 (2015).

11. J. K. Morona, R. Morona, D. C. Miller, J. C. Paton, Mutational Analysis of the Carboxy-Terminal (YGX)(4) Repeat Domain of CpsD, an Autophosphorylating Tyrosine Kinase Required for Capsule Biosynthesis in Streptococcus pneumoniae. J Bacteriol 185, 3009-3019 (2003).

12. J. Yother, Capsules of Streptococcus pneumoniae and other bacteria: paradigms for polysaccharide biosynthesis and regulation. Annu Rev Microbiol 65, 563-581 (2011).

13. M. X. Henriques, T. Rodrigues, M. Carido, L. Ferreira, S. R. Filipe, Synthesis of capsular polysaccharide at the division septum of Streptococcus pneumoniae is dependent on a bacterial tyrosine kinase. Mol Microbiol 82, 515-534 (2011).

14. N. Mirouze, J.-P. Claverys, P. Noirot, S. Université Paul, [s.n.], [S.I.] (2007).

15. K. Yamanaka, T. Ogura, H. Niki, S. Hiraga, Identification of two new genes, mukE and mukF, involved in chromosome partitioning in Escherichia coli. Mol Gen Genet 250, 241-251 (1996).

16. J. Slager, M. Kjos, L. Attaiech, J. W. Veening, Antibiotic-induced replication stress triggers bacterial competence by increasing gene dosage near the origin. Cell 157, 395-406 (2014).

17. M. V. Francia, K. E. Weaver, P. Goicoechea, P. Tille, D. B. Clewell, Characterization of an active partition system for the Enterococcus faecalis pheromone-responding plasmid pAD1. J Bacteriol 189, 8546-8555 (2007).

18. R. van Raaphorst, M. Kjos, J. W. Veening, Chromosome segregation drives division site selection in Streptococcus pneumoniae. Proc Natl Acad Sci U S A 114, E5959-E5968 (2017).

19. H. Zhou, J. Lutkenhaus, Membrane binding by MinD involves insertion of hydrophobic residues within the C-terminal amphipathic helix into the bilayer. J Bacteriol 185, 4326-4335 (2003).

20. L. Aravind, V. Anantharaman, S. Balaji, M. M. Babu, L. M. Iyer, The many faces of the helix-turn-helix domain: transcription regulation and beyond. FEMS Microbiol Rev 29, 231-262 (2005).

21. D. D. Leipe, Y. I. Wolf, E. V. Koonin, L. Aravind, Classification and evolution of P-loop GTPases and related ATPases. J Mol Biol 317 41-72 (2002).

22. K. Gerdes, M. Howard, F. Szardenings, Pushing and pulling in prokaryotic DNA segregation. Cell 141, 927-942 (2010). 
bioRxiv preprint doi: https://doi.org/10.1101/359943; this version posted July 3, 2018. The copyright holder for this preprint (which was not certified by peer review) is the author/funder, who has granted bioRxiv a license to display the preprint in perpetuity. It is made available under aCC-BY-NC-ND 4.0 International license.

23. A. Fleurie et al., Mutational dissection of the S/T-kinase StkP reveals crucial roles in cell division of Streptococcus pneumoniae. Mol Microbiol 83, 746-758 (2012).

24. K. Beilharz et al., Control of cell division in Streptococcus pneumoniae by the conserved Ser/Thr protein kinase StkP. Proc Natl Acad Sci U S A 109, E905-913 (2012).

25. W. O. Bullock, J. M. Fernandez, J. M. short, a high efficiency plasmid transforming recA Escherichia coli strain with betagalactosidase selection. Biotechniques 5, 376 (1987).

26. F. W. Studier, B. A. Moffatt, Use of bacteriophage T7 RNA polymerase to direct selective high-level expression of cloned genes. J Mol Biol 189, 113-130 (1986).

27. C. K. Sung, H. Li, J. P. Claverys, D. A. Morrison, An rpsL cassette, janus, for gene replacement through negative selection in Streptococcus pneumoniae. Appl Environ Microbiol 67, 5190-5196 (2001).

28. K. H. Berg, T. J. Biornstad, D. Straume, L. S. Havarstein, Peptideregulated gene depletion system developed for use in Streptococcus pneumoniae. J Bacteriol 193, 5207-5215 (2011).

29. J. C. Cortay et al., In vitro asymmetric binding of the pleiotropic regulatory protein, FruR, to the ace operator controlling glyoxylate shunt enzyme synthesis. J Biol Chem 269, 14885-14891 (1994).

30. E. Marchadier et al., An expanded protein-protein interaction network in Bacillus subtilis reveals a group of hubs: Exploration by an integrative approach. Proteomics 11, 2981-2991 (2011).

31. P. James, J. Halladay, E. A. Craig, Genomic libraries and a host strain designed for highly efficient two-hybrid selection in yeast. Genetics 144, 1425-1436 (1996).
32. I. G. de Jong, K. Beilharz, O. P. Kuipers, J. W. Veening, Live Cell Imaging of Bacillus subtilis and Streptococcus pneumoniae using Automated Time-lapse Microscopy. J Vis Exp, (2011).

33. A. Ducret, E. M. Quardokus, Y. V. Brun, MicrobeJ, a tool for high throughput bacterial cell detection and quantitative analysis. Nat Microbiol 1, 16077 (2016).

34. M. Jerabek-Willemsen, C. J. Wienken, D. Braun, P. Baaske, S. Duhr, Molecular interaction studies using microscale thermophoresis. Assay Drug Dev Technol 9, 342-353 (2011).

35. K. J. Livak, T. D. Schmittgen, Analysis of relative gene expression data using real-time quantitative PCR and the 2(-Delta Delta C(T)) Method. Methods 25, 402-408 (2001).

36. K. Katoh, D. M. Standley, MAFFT multiple sequence alignment software version 7: improvements in performance and usability. Mol Biol Evol 30, 772-780 (2013).

37. S. R. Eddy, A new generation of homology search tools based on probabilistic inference. Genome Inform 23, 205-211 (2009).

38. A. Criscuolo, S. Gribaldo, BMGE (Block Mapping and Gathering with Entropy): a new software for selection of phylogenetic informative regions from multiple sequence alignments. BMC Evol Biol 10, 210 (2010).

39. S. Guindon et al., New algorithms and methods to estimate maximum-likelihood phylogenies: assessing the performance of PhyML 3.0. Syst Biol 59, 307-321 (2010).

40. D. T. Jones, Protein secondary structure prediction based on position-specific scoring matrices. J Mol Biol 292, 195-202 (1999). 